\title{
Rapid protocol for irinotecan desensitization: a case report and literature review
}

\author{
Gloria Andriollo · Sara Urbani · Alessandro Buonomo · Arianna Aruanno · Eleonora Nucera
}

Received: 31 August 2020 / Accepted: 3 September 2020 / Published online: 10 December 2020

(C) The Author(s) 2020

Keywords Irinotecan · Desensitization • Hypersensitivity · Immediate reaction . Chemotherapy safety

$\begin{array}{ll}\text { Abbreviations } \\ \text { BTR } & \text { Breakthrough adverse reactions } \\ \text { CT } & \text { Computed tomography } \\ \text { HSR } & \text { Hypersensitivity reaction } \\ \text { RDD } & \text { Rapid drug desensitization }\end{array}$

\section{Background}

Irinotecan - an antineoplastic drug that inhibits topoisomerase I-is widely used to treat gastrointestinal malignancies. IgE-mediated hypersensitivity reactions (HSRs) to irinotecan are rarely described to date and successfully treated by a rapid drug desensitization (RDD) protocol. Chemotherapy desensitization is reported as a safe and effective way to reintroduce a culprit drug that often represents the firstline drug therapy. RDD should always be considered, particularly when there are no other valid therapeutic approaches in terms of life expectancy and quality of life. The RDD induces a temporary tolerance to drug allergens by gradually reintroducing a small amount

Consent to participate: we receive consent for publication Consent for publication: we receive consent for publication The authors Gloria Andriollo and Sara Urbani contributed equally to this work.

G. Andriollo, MD · S. Urbani, MD · A. Buonomo, MD ·

A. Aruanno, $\mathrm{MD}, \mathrm{PhD}$

Allergy Unit, Fondazione Policlinico Universitario A. Gemelli

IRCCS, Largo F. Vito, 1, 00168 Rome, Italy

E. Nucera, MD $(\varangle)$

Università Cattolica del Sacro Cuore, Largo F.

Vito, 1, 00168 Rome, Italy

eleonora.nucera@unicatt.it of the antigen over a relatively short period of time until the total scheduled dose has been administrated.

We present the case of a patient affected by pancreas adenocarcinoma who developed an immediate HSR to irinotecan who unsuccessfully underwent a standard chemotherapy RDD protocol.

\section{Case report}

We report a case of a 46-year-old man affected by locally advanced pancreas adenocarcinoma. Neoadjuvant chemotherapy was administered with the FOLFIRINOX regimen (calcium levofolinate, fluorouracil, irinotecan and oxaliplatin) as first-line treatment. During the second administration of irinotecan, the patient presented dyspnea with labial and eyelid angioedema. Subsequently, the patient received a third dose of irinotecan with a slower infusion rate and with a previous corticosteroids dose reporting the same symptoms; he tolerated fluorouracil and oxaliplatin administration and continued with only FOLFOX regimen.

Considering that after 3 months the computed tomography (CT) showed local cancer progression and blood tests revealed an increase of tumor markers (Ca 19.9, CEA), the oncologist team decided to obtain a patient allergological evaluation in order to treat the patient with FOLFIRI regimen (5-fluorouracil, leucovorin, irinotecan).

Hence, we performed irinotecan prick test $(20 \mathrm{mg} / \mathrm{mL})$ and intradermal tests $(2 \mathrm{mg} / \mathrm{mL}$ and $20 \mathrm{mg} / \mathrm{mL}$ ) according to the indications of AlvarezCuesta et al. [1]. The $20 \mathrm{mg} / \mathrm{mL}$ intradermal test was positive (mean wheal of $6 \mathrm{~mm}$ with erythema). On the basis of this result, we diagnosed an irinotecan IgEmediated allergy.

Since the patient needed irinotecan to treat his disease, we proposed irinotecan desensitization using 
Table 1 Main paper on irinotecan hypersensitivity

\begin{tabular}{|c|c|c|c|c|c|c|c|}
\hline Study—first author, year [ref.] & $\begin{array}{l}\text { Age } \\
\text { (years) }\end{array}$ & Gender & Neoplastic disease & Reactions $^{\mathrm{a}}$ & Skin tests (SPT/IDT) & $\begin{array}{l}\text { Desensitization } \\
\text { protocol }\end{array}$ & Outcome \\
\hline Abu-Amna et al., 2015 [6] & 39 & Female & $\begin{array}{l}\text { Metastatic colorectal } \\
\text { cancer }\end{array}$ & Severe & Positive $(0.2 \mathrm{mg} / \mathrm{ml})$ & \multirow{3}{*}{$\begin{array}{l}12 \text { steps protocol } \\
\text { adapted or according } \\
\text { to Castells et al. [4] }\end{array}$} & \multirow[t]{3}{*}{$\begin{array}{l}\text { Successful } \\
\text { RDD }\end{array}$} \\
\hline Cubero et al., 2016 [7] & 57 & Male & Low rectal cancer & Severe & Positive (20 mg/ml) & & \\
\hline Kendirlinan R et al., 2019 [8] & 35 & Female & Colorectal cancer & Moderate & ND & & \\
\hline
\end{tabular}

a 12-step protocol adapted from Castells et al. [2] after premedication with corticosteroid and antihistamines (oral methylprednisolone $32 \mathrm{mg}$ and cetirizine $10 \mathrm{mg}$ from $48 \mathrm{~h}$ before and intravenous methylprednisolone $40 \mathrm{mg}$ and chlorpheniramine $1 \mathrm{~h}$ before the infusion). The irinotecan was diluted in $250 \mathrm{~mL}$ of saline solution and administered 100-fold diluted in solution A, 10 -fold diluted in solution $B$ and the concentration of solution $\mathrm{C}$ was calculated by subtracting the cumulative. At each step, the infusion rate was increased. Solutions were administered in an oncology service by well-trained nursing staff under allergist supervision.

The patient tolerated the first cycle of desensitization therapy well, but during the second cycle he experienced severe systemic symptoms characterized by dyspnea and wheezing that lead to interruption of treatment.

Therefore, we decided to modify the desensitization protocol adding a further step with more diluted drug and increasing premedication with montelukast $10 \mathrm{mg}$. However, due to the severity of HSRs and considering the patient's clinical condition, the oncology team refused this new treatment approach.

\section{Discussion}

HSRs to a chemotherapeutic agent is defined as an unpredictable reaction whose signs and symptoms cannot be explained by toxicity of the drug and range from mild cutaneous manifestation to life-threatening anaphylaxis [3].

HSRs are increased in oncology patients, and their frequency has been reported to be $5-27 \%$ for platins, $10-30 \%$ for taxanes and $0.6-10 \%$ for specific monoclonal antibodies [4]. However, HSRs to irinotecan are less frequently observed and described to date. In the study of Alvarez Cuesta et al. [1], irinotecan was the suspected culprit drug in 11 of 186 patients $(5.9 \%)$ referred to their department for desensitization over a 3-year period.

In case of HRS, the RDD protocol represents a standard of care to continue the necessary chemotherapy drugs when no alternative equally effective therapy is available. Indeed RDD induces a temporary toleration state through incremental administration of drug. Although the molecular basis of this desensitization remains incompletely understood, the most likely hypothesis is that RDD inhibits mast cell activation.
However, as in our case, the occurrence of breakthrough adverse reactions (BTRs) during RDD are reported in $10-40 \%$ of cases $[2,5]$ and their severity is significantly associated with initial HSRs severity, history of drug allergy and previous exposure to chemotherapeutic agents. The BTRs, instead, do not seem to be related to type and dose of premedication.

In the literature, only three cases of irinotecan RDD are described without any reaction during the protocol unlike our case (Table $1 ;$ [6-8]).

These protocols differed only in terms of premedication; in the first case, it consisted only of corticosteroids and antihistamine therapy, while, in the second one, the pretreatment included oral $500 \mathrm{mg}$ acetylsalicylic acid and oral $10 \mathrm{mg}$ montelukast at $48 \mathrm{~h}$ and $24 \mathrm{~h}$ before and on the day of desensitization. This latter approach seems be associated with a reduction of BTR occurrence in comparison to methylprednisolone pretreatment, considering the role of prostaglandins D2 and leukotrienes in the mast cell activation [10].

In our case, the oncologist team stopped irinotecan therapy after the first BRT although it was not worse than the initial reaction without trying implementation of proposed premedication and it lead to an unsuccessfully RDD. For this reason, consensus between oncologist and allergist in the assessment and management of HSRs and RDD is always required in order to avoid discontinuation of chemotherapeutic drugs.

On the basis of our experience and variable irinotecan RDD outcome, more studies are needed to propose individualized premedications and desensitization protocols according to patient-specific risk stratification.

\section{Conclusions}

The irinotecan RDD protocol should be always carried out taking into consideration the "risk-benefit balance" in an appropriate setting and mainly applied to patients who have no other reasonable alternative, but have the obvious potential clinical benefit of further treatment with the culprit drug.

Thus, the fear of more severe reaction during irinotecan infusion should not lead the clinicians to interrupt the ongoing chemotherapic therapy. Indeed, RDD should be always evaluated as an individualized possible therapeutic route in patients with HSRs; con- 
sidering that it has a certain degree of risk, it should be performed in a specialized and safe setting under the close supervision of professional care from different disciplines.

Acknowledgements The authors are responsible for the content and the writing of this paper.

Funding The authors declare that no funding was received for the present case report.

Funding Open access funding provided by Università Cattolica del Sacro Cuore within the CRUI-CARE Agreement.

Open Access This article is licensed under a Creative Commons Attribution 4.0 International License, which permits use, sharing, adaptation, distribution and reproduction in any medium or format, as long as you give appropriate credit to the original author(s) and the source, provide a link to the Creative Commons licence, and indicate if changes were made. The images or other third party material in this article are included in the article's Creative Commons licence, unless indicated otherwise in a credit line to the material. If material is not included in the article's Creative Commons licence and your intended use is not permitted by statutory regulation or exceeds the permitted use, you will need to obtain permission directly from the copyright holder. To view a copy of this licence, visit http://creativecommons.org/licenses/by/4.0/.

\section{References}

1. Alvarez-Cuesta E, Madrigal-Burgaleta R, Angel-Pereira D, Ureña-Tavera A, Zamora-Verduga M, Lopez-Gonzalez $P$, et al. Delving into cornerstones of hypersensitivity to antineoplastic and biological agents: value of diagnostic tools prior to desensitization. Allergy. 2015;70:784-94.
2. Castells MC, Tennant NM, Sloane DE, Hsu FI, Barrett NA, Hong DI, et al. Hypersensitivity reactions to chemotherapy: outcomes and safety of rapid desensitization in 413 cases. JAllergy Clin Immunol. 2008;122:574-80.

3. Syrigou E, Makrilia N, Koti I, Saif MW, Syrigos KN. Hypersensitivity reactions to antineoplastic agents: an overview. Anticancer Drugs. 2009;20:1-6.

4. Castells Guitart MC. Rapid drug desensitization for hypersensitivity reactions to chemotherapy and monoclonal antibodies in the 21 st century. J Investig Allergol Clin Immunol. 2014;24:72-9.

5. Kang Y, Kwon OY, Jung H, Kang M, An J, Lee J, et al. Breakthrough reactions during rapid drug desensitization: clinical outcome and risk factors. Ann Allergy Asthma Immunol. 2019;123:48-56:e1.

6. Abu-Amna M, Hassoun G, Hadad S, Haim N, Bar-Sela G. Successful desensitization protocol for hypersensitivity reaction caused by Irinotecan in a patient with metastatic colorectal cancer. Clin Colorectal Cancer. 2015;14:e49-e51.

7. Cubero JL, Escudero P, Yubero A, Millán P, Sagredo MA, Colás C. Successful desensitization to Irinotecan after severe hypersensitivity reaction. J Investig Allergol Clin Immunol. 2016;26:314-6.

8. Kendirlinan R, Gümüşburun R, Çerçi P, Özbek E, Altıner S, Sözener ZC, et al. Rapid drug desensitization with chemotherapeutics (Platins, Taxanes, and others): a single-center retrospective study. Int Arch Allergy Immunol. 2019;179:114-22.

9. Brown SG. Clinical features and severity grading of anaphylaxis. JAllergy Clin Immunol. 2004;114:371-6.

10. Breslow RG, Caiado J, Castells MC. Acetylsalicylic acid and montelukast block mast cell mediator-related symptoms during rapid desensitization. Ann Allergy Asthma Immunol. 2009;102:155-60. 